\title{
The Lkb1 metabolic sensor maintains haematopoietic stem cell survival
}

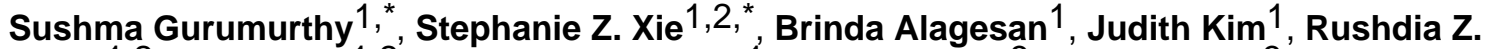 \\ Yusuf $^{1,2}$, Borja Saez ${ }^{1,2}$, Alexandros Tzatsos ${ }^{1}$, Fatih Ozsolak ${ }^{3}$, Patrice Milos ${ }^{3}$, Francesco \\ Ferrari $^{4}$, Peter J. Park ${ }^{4}$, Orian S. Shirihai ${ }^{5}$, David T. Scadden ${ }^{1,2}$, and Nabeel Bardeesy ${ }^{1}$ \\ ${ }^{1}$ Cancer Center and Center for Regenerative Medicine, Massachusetts General Hospital and \\ Harvard Medical School, Boston, Massachusetts 02114, USA \\ ${ }^{2}$ Harvard Stem Cell Institute and Department of Stem Cell and Regenerative Biology, Harvard \\ University, Boston, Massachusetts 02115, USA \\ ${ }^{3}$ Helicos BioSciences Corporation, Cambridge, Massachusetts 02139, USA \\ ${ }^{4}$ Center for Biomedical Informatics and Informatics Program, Children's Hospital, and Harvard \\ Medical School, Boston, Massachusetts 02115, USA \\ ${ }^{5}$ Department of Medicine, Evans Research Center, Mitochondria ARC, Boston University Medical \\ Center, Boston, Massachusetts 02118, USA
}

\begin{abstract}
Haematopoietic stem cells (HSCs) can convert between growth states that have marked differences in bioenergetic needs. Although often quiescent in adults, these cells become proliferative upon physiological demand. Balancing HSC energetics in response to nutrient availability and growth state is poorly understood, yet essential for the dynamism of the haematopoietic system. Here we show that the Lkb1 tumour suppressor is critical for the maintenance of energy homeostasis in haematopoietic cells. $L \mathrm{kbl}$ inactivation in adult mice causes loss of HSC quiescence followed by rapid depletion of all haematopoietic subpopulations. Lkb1deficient bone marrow cells exhibit mitochondrial defects, alterations in lipid and nucleotide metabolism, and depletion of cellular ATP. The haematopoietic effects are largely independent of Lkb1 regulation of AMP-activated protein kinase (AMPK) and mammalian target of rapamycin (mTOR) signalling. Instead, these data define a central role for $L k b l$ in restricting HSC entry into cell cycle and in broadly maintaining energy homeostasis in haematopoietic cells through a novel metabolic checkpoint.
\end{abstract}

The metabolic control systems in HSCs are poorly understood although these cells have been shown to be highly sensitive to energetic and oxidative stress and must be able to shift

\footnotetext{
(c) 2010 Macmillan Publishers Limited. All rights reserved

Correspondence and requests for materials should be addressed to N.B. (Bardeesy.Nabeel@MGH.Harvard.edu) or D.T.S. (Scadden.David@MGH.Harvard.edu).

These authors contributed equally to this work.

Supplementary Information is linked to the online version of the paper at www.nature.com/nature.

Author Contributions S.G. and S.Z.X. performed the experiments with assistance from B.A., J.K., A.T., B.S. and R.Z.Y. S.G., S.Z.X., D.T.S. and N.B. designed the experiments, analysed and evaluated all data, and wrote the manuscript. F.O. and P.M. performed the RNA sequencing. F.F. and P.J.P. analysed the RNA sequencing data. O.S.S. designed and evaluated the oxygen consumption experiments.

Author Information Reprints and permissions information is available at www.nature.com/reprints. The authors declare no competing financial interests. Readers are welcome to comment on the online version of this article at www.nature.com/nature.
} 
between quiescence and highly proliferative states to maintain the haematopoietic system under varying physiological conditions ${ }^{1-5}$. The $L k b l$ tumour suppressor encodes an evolutionarily conserved serine/threonine kinase that links metabolism and cell growth ${ }^{6}$. The most clearly defined physiological function of Lkb1 is to phosphorylate and activate AMPK in response to a decline in the cellular energy charge (ATP/ AMP ratio) $)^{7}, 8$. AMPK restores cellular ATP levels through phosphorylation of key regulatory proteins involved in protein synthesis, fatty acid and glucose metabolism, and glucose transport9. This results in inhibition of energy expending processes and promotes ATP generation. In Caenorhabditis elegans, an Lkb1-AMPK pathway is critical in inducing quiescence in germline stemcell populations ${ }^{10}$. Lkb1 can also regulate energy homeostasis and cell structure through its capacity to phosphorylate and activate 12 other AMPK-related kinases ${ }^{11}$. Given the importance ofHSCdynamics to the haematopoietic system and the sensitivity of haematopoietic cells to metabolic stress, we sought to investigate the function of $L k b l$ in haematopoiesis.

\section{Lkb1 deletion causes bone marrow failure}

Lkbl mRNA expression is readily detectable in haematopoietic cells in the bone marrow, with highest levels in $\mathrm{HSCs}^{12}$ and in multipotent and lineage-restricted progenitors compared to committed $\mathrm{Lin}^{+}$cells (Supplementary Fig. 1a). To study Lkbl function in haematopoiesis, we bred conditional $L k b I^{L / L}$ mice (ref. 13) with the Mxl-cre strain $^{14}$ and induced Cre recombinase activity by administration of polyinosinicpolycytidylic acid (pIpC). Experimental $M x l$-cre; $L k b 1^{L / L}$ mice (designated $L k b 1$ mutants) showed a rapid decrease in bone marrow cellularity and $93 \%$ died within 30 days after pIpC induction (Fig. 1a, b); Mxl-cre; $L k b 1^{L /+}$ and Mxl-cre; $L k b 1^{+/+}$mice showed normal survival and bone marrow cellularity and were used as controls. Analysis of the bone marrow and individual haematopoietic lineages demonstrated efficient $L k b 1$ deletion, marked reductions in Lkb1 polypeptide levels, reduced AMPK activity without changes in total AMPK $\alpha$ levels, and increased mTOR complex I (mTORC1) activity in the Lkbl mutants (Supplementary Fig. $1 b-e)$.

Lkb1 mutant animals displayed progressive pancytopenia as well as rapid loss of bone marrow myeloid, B lymphoid and erythroid cells (Fig. 1c, d and Supplementary Fig. 2a), and markedly decreased cellularity of the thymus and spleen (Supplementary Fig. 2c, d and data not shown). Notably, in the bone marrow and thymus, immature lymphoid cells declined at a faster rate than the more differentiated cells (Supplementary Fig. 2b, c). The Lkbl mutants also exhibited a pronounced loss of HSC and multipotent progenitor populations at day 5 after pIpC treatment (Fig. 1e, f and Supplementary Fig. 2f). Furthermore, $L k b 1$ mutant bone marrow cells formed fewer and smaller colonies in in vitro colony forming assays (Fig. 1g and Supplementary Fig. 2e). Comparable in vivo and in vitro phenotypes were seen using a second model system in which $L k b l$ was deleted using the tamoxifen-inducible Rosa26-creERt2 strain (Supplementary Fig. 2g, h, and not shown). These results show that $L k b l$ is critically required for haematopoiesis and for the maintenance of HSCs and progenitor cells.

\section{Lkb1 function in bone marrow is cell intrinsic}

Both theMxl-cre and Rosa26-creERt2 systems induce Cre recombinase activity in many cell types; thus bone marrow transplants were used to determine whether $L k b l$ has a cellautonomous role in haematopoiesis. We performed non-competitive transplants of CD45.1 whole bone marrow from control or $M x I$-cre; $L k b I^{L / L}$ mice into lethally irradiated CD 45.2 wild-type congenic recipients, confirmed stable reconstitution, and then administered pIpC (Supplementary Fig. 3a). The results showed that mice transplanted with $L k b 1$ mutant cells 
died within 12 weeks and exhibited acute pancytopenia and rapid decline of $L k b 1$ mutant donor cells in the peripheral blood (Fig. 2a and Supplementary Fig. 3b, c). The effects on bone marrow cells were even more pronounced; there was a marked decrease in cellularity and chimaerism as early as day 5 (Fig. $2 \mathrm{~b}$ ) and the $L k b l$ mutant donor stem cell, progenitor andmature populations were largely depleted by day 18 (Fig. 2c, and not shown). We performed reciprocal transplant experiments in which wild-type donor cells were transplanted into either control or $L k b l$ mutant recipients; treatment with $\mathrm{pIpC}$ after stable reconstitution did not result in any alterations in haematopoiesis in the $L k b 1$ mutant recipients (Supplementary Fig. 4).

To extend these studies, we transplanted donor CD45.1 control or Mxl-cre; $L k b 1^{L / L}$ bone marrow cells with a 1:1 ratio of competitor CD45.2 wild-type bone marrow cells into CD45.2 recipient mice (Supplementary Fig. 3d). After engraftment, the peripheral blood of mice transplanted with $M x I$-cre; $L k b I^{L / L}$ bone marrow cells had a lower rate of chimaerism compared to controls (30\% versus 53\%) (Fig. 2d), an effect probably due to the induction of interferon associated with transplant ${ }^{15,16}$. Nonetheless, upon $\mathrm{pIpC}$ administration, $M x 1$-cre; $L k b 1^{L / L}$ transplanted recipients displayed a sharp decline in CD45.1 cells in the peripheral blood (Fig. 2d and Supplementary Fig. 3e, f). Moreover, there was a complete absence of Lkb1 mutant CD45.1 HSCs in the bone marrow 4 weeks after induction (Fig. 2e and not shown). Hence, there is an absolute and intrinsic requirement for $L k b l$ in HSCs and in the maintenance of haematopoiesis.

\section{Lkb1 maintains HSC quiescence}

Defects in proliferative control can contribute to HSC exhaustion. To study the acute impact of $L k b 1$ inactivation on the absolute number and cell cycle profiles of HSCs, we analysed the bone marrow after administering a shorter course of pIpC injections (designated day-3), as well as at a slightly later time point (day +2 ) (Supplementary Fig. 5a).Notably, Lkblmutants showed 2.5-fold increase in HSCs at day -3 , whereas they were markedly reduced at all subsequent time points (Fig. 3a; also see Fig. 1e). A comparable profile was seen in the Rosa26-creERt 2 model (Supplementary Figs $5 \mathrm{~b}$ and $2 \mathrm{~h}$ ). This early increase in cell number was restricted to HSCs among all the bone marrow subpopulations (not shown).

To assess whether the early, transient increase in HSC number was associated with enhanced proliferation, we performed cell cycle analysis using the proliferation marker $\mathrm{Ki}-67$ and propidium iodide to mark DNA content. pIpC treatment has been previously shown to induce G1 cell-cycle entry in wild-type $\operatorname{HSCs}^{17}$ and, as expected, we found that this treatment increased the proportion of Ki-67-positive cells (Fig. 3b). Nonetheless, similarly low percentages of proliferating HSCs in S/G2/M were observed in the untreated mice and pIpC-treated controls (6.9\% and $7.0 \%$ respectively), whereas $>21 \%$ of mutant HSCs were cycling in S/G2/M (Supplementary Fig. 5c). Smaller, but still significant, increases in HSC proliferation rates were observed at later time points in the $L k b 1$ mutants, despite the decline in HSC numbers (Supplementary Fig. 5c). Comparable results were observed using the Rosa26-creERt2 system (Supplementary Fig. 5d). Lkb1 inactivation had a more modest effect on the cell-cycle profiles of differentiated $\mathrm{Lin}^{+}$cells (Fig. 3b, bottom panel). Thus, $L k b 1$ is required for the maintenance of HSC quiescence and its loss causes bone marrow failure that is preceded by an increase in the proliferation and absolute number of HSCs.

\section{Lkb1 loss induces apoptosis and autophagy}

We subsequently assessed the impact of $L k b l$ loss on cell death pathways in haematopoietic cells. $L k b 1$ mutant cells had reduced viability by day 5 after $\mathrm{pIpC}$ as documented by 7amino-actinomycinD(7AAD) staining; stem and progenitor cells exhibited more pronounced 
sensitivity compared to more differentiated cells (Fig. 3c and Supplementary Fig. 5e, f). Annexin V/7AAD analysis demonstrated that apoptosis was induced in the $L k b 1$ mutant HSCs and progenitors starting at day 3 and elevated $\sim$-fold by day 5 ; similar profiles were seen in Mac- $1^{+}$and B220 $0^{+}$cells (Supplementary Fig. 5f). Correspondingly, lysates from Lkb1 mutant bone marrow, spleen and thymus exhibited an elevation in cleaved caspase- 3 levels starting at day 3 after pIpC (Fig. 3d).

Next, we investigated the cellular stress responses that may underlie the cell death phenotypes. Macroautophagy (autophagy) is a process of organelle and long-lived protein degradation that is activated in response to nutrient deprivation or oxidative stress and can either serve as a survival mechanism or promote cell death depending on cellular context ${ }^{18}$. The lipidated form of the LC3 protein, LC3-II, serves as a marker for active autophagy19. Notably, immunoblot analysis at day 1 after pIpC treatment showed that LC3-II levels were elevated in $L k b 1$ mutant bone marrow, thymus and spleen (Fig. 3e). In support of these findings, immunofluorescence staining demonstrated a fivefold increase in the proportion of bone marrow cells showing LC3 puncta in the Lkb1 mutants (Supplementary Fig. 5g). Treatment of mice with chloroquine, which inhibits autophagy by blocking lysosomal acidification and autophagosome degradation, accelerated the death of the $L \mathrm{kbl}$ mutants while not affecting control mice (not shown), indicating that autophagy induction served as a survival response in these cells. $L k b 1$ mutant bone marrow also exhibited increased expression of phospho(Ser 139)-histone H2AX (phospho-H2AX), and a threefold increase in phospho-H2AX foci, indicating the presence of ongoing DNA damage (Fig. 3f and Supplementary Fig. 5h). Hence, apoptotic cell death is markedly increased in Lkbl mutant haematopoietic cells and may be associated with nutrient and genotoxic stress.

\section{Lkb1 function is AMPK/mTORC1-independent}

Inactivation of mTORC1 is a major component of the Lkb1-AMPK energy stress response in primary fibroblasts and cancer cell lines ${ }^{20,21}$. In addition, knockout of the mTORC1 regulator, $T s c 1$, results in bone marrow failure due to mTORC1 activation and induction of reactive oxygen species $(\mathrm{ROS})^{5}$. $\mathrm{Lkbl}$ inactivation led to an increase in mTORC1 activity in whole bone marrow as reflected by phospho (serine-235/236)-ribosomal protein S6 levels; yet analysis of bone marrow subpopulations revealed that this increase was entirely due to changes in myeloid cells (Fig. 4a and Supplementary 6a) - a finding in contrast to Tscl and $T s c 2$ deficiency, which results in mTORC1 activation in all haematopoietic cells ${ }^{2,5}$.

Correspondingly, inhibition of mTORC1 by administration of rapamycin failed to restore bone marrow cellularity or the declines in individual populations in the $L k b 1$ mutants despite suppressing S6 phosphorylation (Fig. 4b, c and Supplementary Fig. 6b-d). Administration of the anti-oxidant $N$-acetyl-cysteine (NAC) also had no effect on lifespan or bone marrow cellularity (Supplementary Fig. 6e, f and data not shown). Therefore, deregulation of the mTORC1 pathway does not underlie the haematopoietic defects in these mice.

AMPK regulates cell metabolism through multiple pathways in addition to mTORC1. Because AMPK activity is eliminated upon $L k b 1$ deletion in the bone marrow (Supplementary Fig. 1d, e), we sought to test whether the allosteric AMPK activator, A-769662, rescues the haematopoietic defects in these mice. A-769662 induced (Ser 79)acetyl-CoA carboxylase (ACC) phosphorylation in primary fibroblasts in an AMPK $\alpha 1 / \alpha 2$ dependent, but $L k b 1$-independent, manner (Supplementary Fig. 6g). When administered before and throughout the course of the pIpC treatment in the Mxl-cre model, this compound also effectively restored AMPK activity in $L k b l$-deficient bone marrow; however, there was no rescue of any bone marrow subpopulations or of overall survival (Fig. 4d-f and Supplementary Fig. 6h and not shown). Hence AMPK may not be a critical effector of Lkb1 in the maintenance of haematopoiesis. 


\section{Lkb1 maintains bone marrow energy homeostasis}

Lkbl deficiency in liver and muscle leads to broad alterations in energy usage, lipid and carbohydrate metabolism and mitochondrial mass. These effects are only partially AMPK dependent, and probably also reflect roles of additional AMPK-related kinases ${ }^{22-24}$. Therefore, we assessed whether the $L k b l$ mutant bone marrow exhibited metabolic defects, as indicated by our LC 3 and chloroquine data. Notably, there was a marked decline in mitochondrial membrane potential in the $L k b 1$ mutant common myeloid progenitors (CMPs), common lymphoid progenitors (CLPs) and HSCs at day 3 after pIpC treatment (Fig. 5a). These alterations were associated with defects in bioenergetics, as basal mitochondrial oxygen consumption and total mitochondrial oxidative capacity were reduced in the $L k b 1$ mutants at day 1 after induction in both models (Fig. 5b); the use of a shortened induction scheme confirmed that these changes occurred rapidly upon $L k b 1$ deletion (Supplementary Fig. 7a). Consistent with a broad inability to maintain energy homeostasis, lineage-negative bone marrow cells had a significant decline in basal ATP levels by day 5 , despite showing increased glucose uptake (Fig. 5c, d and Supplementary 7b). Mitochondrial content was significantly decreased upon initial $L k b l$ inactivation whereas it was elevated at later time points ( $>5$ days after pIpC) (Fig. 5e and Supplementary Fig. $7 \mathrm{~b}$ ); this biphasic effect seems to reflect a compensatory response to the metabolic alterations in these cells. Collectively, these data indicate that $L k b l$ mutant haematopoietic cells are unable to sustain the cellular energy charge and have defects in mitochondrial function.

To define the metabolic alterations in $L k b 1$-deficient cells further, we evaluated the global metabolic profiles of both differentiated $\left(\mathrm{Lin}^{+}\right)$and lineage negative $\left(\mathrm{Lin}^{-}\right)$populations at day 1 after $\mathrm{pIpC}$ using a combination of liquid/gas chromatography and mass spectrometry (GC/MS and LC/MS/MS) platforms. This analysis showed that the Lkb1 mutants had statistically significant alterations in lipid metabolism in the $\mathrm{Lin}^{+}$and $\mathrm{Lin}^{-}$populations (specifically increases in most detectable long-chain fatty acids and in several essential fatty acids and elevations in nucleotide metabolites (only in $L k b 1$ mutant $\mathrm{Lin}^{-}$cells); Supplementary Figs 8 and 9). Individual amino acids, dipeptides and some glycolysis and citric acid cycle components showed differences; although, when considered as groups, these metabolites were not altered in our analysis (Supplementary Fig. 10 and not shown). The diminished mitochondrial function and elevations in fatty acid levels are consistent with a model whereby Lkb1 serves as a rheostat that sets the appropriate balance of anabolic and catabolic activities in haematopoietic cells.

\section{Discussion}

The extreme sensitivity of haematopoietic cells to $L k b l$ inactivation is remarkable when compared to the impact of $L k b l$ deficiency in other cell types. In particular, $L k b l$ deficiency results in the development of solid tumours of multiple tissue types and in the immortalization of primary fibroblasts in vitro ${ }^{13,25-28}$. Lkbl inactivation does confer increased sensitivity to several stress states including nutrient deprivation, oxidative stress and hypoxia ${ }^{7,29}$. Here we show that in the haematopoietic system, where stem cell modulation between quiescence and proliferation is necessary to withstand acute physiological stress while preserving long-term regenerative capacity, $L k b l$ has an unanticipated and critical role. $L k b 1$ is needed to maintain haematopoietic cells broadly, with $L k b l$ deletion leading to the death of multiple subpopulations. The processes involved seem to be defects in metabolic homeostasis associated with mitochondrial dysfunction, ATP depletion, a compensatory autophagic response, and rapid apoptotic cell death. Given the speed of the cell death response, it remains important to establish which of the metabolic alterations are direct consequences of $L k b l$ inactivation and which are secondary to the 
induction of apoptosis; the mitochondrial defects in particular could be associated with early stages of an apoptotic program.

HSCs respond to $L k b 1$ deficiency with cell-cycle entry, an effect not seen in other haematopoietic subsets. Whereas Lkb1 function may have a general role in integrating energy sensing and growth control, its unique role in enforcing quiescence of HSCs raises interesting issues of how this population may be particularly responsive to its metabolic environment. Whether this is due to a primary low-energy state in HSCs that activates Lkb1 signalling or the distinct regulation of Lkb1 in these cells is a subject of further study. Notably, Lkb1 seems to act in the haematopoietic system primarily through pathways independent of AMPK. The data therefore point to a novel metabolic checkpoint active in haematopoiesis. In this regard, direct RNA sequencing ${ }^{30}$ of LKS $\left(\mathrm{Lin}-\mathrm{c}-\mathrm{Kit}^{+} \mathrm{Sca}-1^{+}\right.$) and granulocyte-macrophage progenitor (GMP) cells showed that the transcripts encoding a number of AMPK-related kinases are present at comparable or higher amounts than AMPKal (also called Prkaal) and AMPKa2 (also called Prkaa2) (Supplementary Fig. 11). Several of these candidate Lkb1 effectors have overlapping roles in the regulation of metabolism, polarity and mitosis, indicating that they may serve to coordinate energetic states with alterations in cell structure and growth ${ }^{31}$. Our data and two accompanying studies ${ }^{32,33}$ suggest that Lkb1 controls such processes in haematopoiesis and in HSCs in particular. They reveal that Lkb1 function is central to the preservation of a regenerative tissue that is critical for survival.

\section{METHODS SUMMARY}

$L k b 1^{L / L}$ mice $^{13}$ were crossed to $M x l$-cre mice ${ }^{14}$ and backcrossed for six generations onto a C57BL/6 background. pIpC (Amersham) was administered intraperitoneally (i.p.) four times every other day with $8.04 \mathrm{~g} \mathrm{~kg}^{-1}$ body weight. Rosa26-creERT2; $L \mathrm{kbl}^{L / L}$ mice were injected i.p. with $130 \mathrm{mg} \mathrm{kg}^{-1}$ body weight tamoxifen (Sigma) daily for 5 days. $100 \mathrm{mg}$ $\mathrm{kg}^{-1}$ body weight NAC (Sigma) and $4 \mathrm{mgkg}^{-1}$ body weight rapamycin (LC laboratories) were injected i.p. starting 2 days before $\mathrm{pIpC}$ treatment and throughout the course of the experiment. A-769662 (LC laboratories) was administered in drinking water at $30 \mathrm{mg} \mathrm{kg}^{-1}$ of body weight per day. For ROS, mitochondrial number, and membrane potential measurements, bone marrow subpopulations were incubated with dichlorofluorescein diacetate (DCFDA), Mitotracker green and DilC1-5 (Invitrogen), respectively, at $37{ }^{\circ} \mathrm{C}$ for 15-30 min before analysis on the LSRII instrument (Becton, Dickinson). ATP was measured from lysates prepared from 20,000 freshly isolated bone marrow cells with the ATP Bioluminescence Assay Kit HS II (Roche). For repopulation experiments, C57BL/6 (CD45.2) mice were lethally irradiated with $9.5 \mathrm{~Gy}$ and reconstituted by tail vein injections with 1 million bone marrow cells from $M x 1-c r e ; L k b 1^{L / L}$ or control mice. Relative mRNA expression analysis of $L k b l$ and other genes was performed using FastStart SYBR Green Mastermix (Roche) on the MxP3005P real-time PCR system (Stratagene). Analysis of metabolites was performed on $\mathrm{Lin}^{+}$and $\mathrm{Lin}^{-}$bone marrow cells by a combination of liquid/ gas chromatography combined with mass spectrometry (GC/MS and LC/MS/MS) performed by Metabolon Inc. ${ }^{34}$.

Full Methods and any associated references are available in the online version of the paper at www.nature.com/nature.

\section{Supplementary Material}

Refer to Web version on PubMed Central for supplementary material. 


\section{Acknowledgments}

We would like to thank the Harvard Stem Cell Institute Flow Cytometry Core and D. Brown and the MGH Electron Microscope Imaging Core for expertise and advice. V. Levy and M. Leisa provided technical assistance in the SeaHorse measurements. D. Van Buren provided advice and assistance in pilot experiments. We thank A. Camacho for mouse colony assistance. We would also like to thank A. Kimmelman, R. Mostoslavsky, M. Vander Heiden, L. Ellisen, W. Kimand M. Ivan for discussions and critical review of themanuscript.Weare grateful to S. Morrison and R. DePinho for sharing unpublished data. N.B. would like to acknowledge support from NIHU01 CA141576-01. D.T.S would like to acknowledge support from NIH DK050234 and Ellison Medical Foundation. S.G. was supported by a Massachusetts Biotechnology Research Council Tosteson Fellowship. S.Z.X. was supported by an NIH Ruth L. Kirschstein National Research Service Award.

\section{References}

1. Tothova Z, Gilliland DG. FoxO transcription factors and stemcell homeostasis: insights from the hematopoietic system. Cell Stem Cell 2007;1:140-152. [PubMed: 18371346]

2. Gan B, et al. mTORC1-dependent and -independent regulation of stem cell renewal, differentiation, and mobilization. Proc. Natl Acad. Sci. USA 2008;105:19384-19389. [PubMed: 19052232]

3. Ito K, et al. Regulation of reactive oxygen species by Atm is essential for proper response toDNA double-strand breaks in lymphocytes. J. Immunol 2007;178:103-110. [PubMed: 17182545]

4. Liu J, et al. Bmil regulates mitochondrial function and the DNA damage response pathway. Nature 2009;459:387-392. [PubMed: 19404261]

5. Chen C, et al. TSC-mTOR maintains quiescence and function of hematopoietic stem cells by repressing mitochondrial biogenesis and reactive oxygen species. J. Exp. Med 2008;205:23972408. [PubMed: 18809716]

6. Shackelford DB, Shaw RJ. The LKB1-AMPK pathway: metabolism and growth control in tumour suppression. Nature Rev. Cancer 2009;9:563-575. [PubMed: 19629071]

7. Shaw RJ, et al. The tumor suppressor LKB1 kinase directly activates AMP-activated kinase and regulates apoptosis in response to energy stress. Proc. Natl Acad. Sci. USA 2004;101:3329-3335. [PubMed: 14985505]

8. Hawley SA, et al. Complexes between the LKB1 tumor suppressor, STRAD $\alpha / \beta$ and MO $25 \alpha / \beta$ are upstream kinases in the AMP-activated protein kinase cascade. J. Biol 2003;2:28. [PubMed: 14511394]

9. Hardie DG. AMP-activated/SNF1 protein kinases: conserved guardians of cellular energy. Nature Rev. Mol. Cell Biol 2007;8:774-785. [PubMed: 17712357]

10. Narbonne P, Roy R. Caenorhabditis elegans dauers need LKB1/AMPK to ration lipid reserves and ensure long-term survival. Nature 2009;457:210-214. [PubMed: 19052547]

11. Lizcano JM, et al. LKB1 is a master kinase that activates 13 kinases of the AMPK subfamily, including MARK//PAR-1. EMBO J 2004;23:833-843. [PubMed: 14976552]

12. Kiel MJ, et al. SLAM family receptors distinguish hematopoietic stem and progenitor cells and reveal endothelial niches for stem cells. Cell 2005;121:1109-1121. [PubMed: 15989959]

13. Bardeesy N, et al. Loss of the Lkb1 tumour suppressor provokes intestinal polyposis but resistance to transformation. Nature 2002;419:162-167. [PubMed: 12226664]

14. Kuhn R, Schwenk F, Aguet M, Rajewsky K. Inducible gene targeting in mice. Science 1995;269:1427-1429. [PubMed: 7660125]

15. Chan IT, et al. Conditional expression of oncogenic K-ras from its endogenous promoter induces a myeloproliferative disease. J. Clin. Invest 2004;113:528-538. [PubMed: 14966562]

16. Torchia EC, Boyd K, Rehg JE, Qu C, Baker SJ. EWS/FLI-1induces rapidonset of myeloid/ erythroid leukemia in mice. Mol. Cell. Biol 2007;27:7918-7934. [PubMed: 17875932]

17. Essers MAG, et al. IFN $\alpha$ activates dormant haematopoietic stem cells in vivo. Nature 2009;458:904-908. [PubMed: 19212321]

18. Maiuri MC, Zalckvar E, Kimchi A, Kroemer G. Self-eating and self-killing: crosstalk between autophagy and apoptosis. Nature Rev. Mol. Cell Biol 2007;8:741-752. [PubMed: 17717517]

19. Ichimura Y, et al. A ubiquitin-like system mediates protein lipidation. Nature 2000;408:488-492. [PubMed: 11100732] 
20. Shaw RJ, et al. The LKB1 tumor suppressor negatively regulates mTOR signaling. Cancer Cell 2004;6:91-99. [PubMed: 15261145]

21. Corradetti MN, Inoki K, Bardeesy N, DePinho RA, Guan KL. Regulation of the TSC pathway by LKB1: evidence of a molecular link between tuberous sclerosis complex and Peutz-Jeghers syndrome. Genes Dev 2004;18:1533-1538. [PubMed: 15231735]

22. Shaw RJ, et al. The kinase LKB1 mediates glucose homeostasis in liver and therapeutic effects of metformin. Science 2005;310:1642-1646. [PubMed: 16308421]

23. Koh HJ, et al. Skeletal muscle-selective knockout of LKB1 increases insulin sensitivity, improves glucose homeostasis, and decreases TRB3. Mol. Cell. Biol 2006;26:8217-8227. [PubMed: 16966378]

24. Koh HJ, et al. Sucrose nonfermenting AMPK-related kinase (SNARK) mediates contractionstimulated glucose transport in mouse skeletal muscle. Proc. Natl Acad. Sci. USA. 2010 August 16 ;

25. Gurumurthy S, et al. LKB1 deficiency sensitizes mice to carcinogen-induced tumorigenesis. Cancer Res 2008;68:55-63. [PubMed: 18172296]

26. Contreras CM, et al. Loss of Lkb1 provokes highly invasive endometrial adenocarcinomas. Cancer Res 2008;68:759-766. [PubMed: 18245476]

27. Hezel AF, et al. Pancreatic LKB1 deletion leads to acinar polarity defects and cystic neoplasms. Mol. Cell. Biol 2008;28:2414-2425. [PubMed: 18227155]

28. Sanchez-Cespedes M. A role for LKB1 gene in human cancer beyond the Peutz- Jeghers syndrome. Oncogene 2007;26:7825-7832. [PubMed: 17599048]

29. Klimova TA, et al. Hyperoxia-induced premature senescence requires p53 and pRb, but not mitochondrial matrix ROS. FASEB J 2009;23:783-794. [PubMed: 18948382]

30. Ozsolak F, et al. Direct RNA sequencing. Nature 2009;461:814-818. [PubMed: 19776739]

31. Hezel AF, Bardeesy N. LKB1; linking cell structure and tumor suppression. Oncogene 2008;27:6908-6919. [PubMed: 19029933]

32. Nakada D, Saunders TL, Morrison SJ. Lkb1 regulates cell cycle and energy metabolism in haematopoietic stem cells. Nature.

33. Gan B, et al. Lkb1 regulates quiescence and metabolic homeostasis of haematopoietic stem cells. Nature.

34. Watson M, et al. The small molecule GMX1778 is a potent inhibitor of NAD1 biosynthesis: strategy for enhanced therapy in nicotinic acid phosphoribosyltransferase 1-deficient tumors. Mol. Cell. Biol 2009;29:5872-5888. [PubMed: 19703994] 

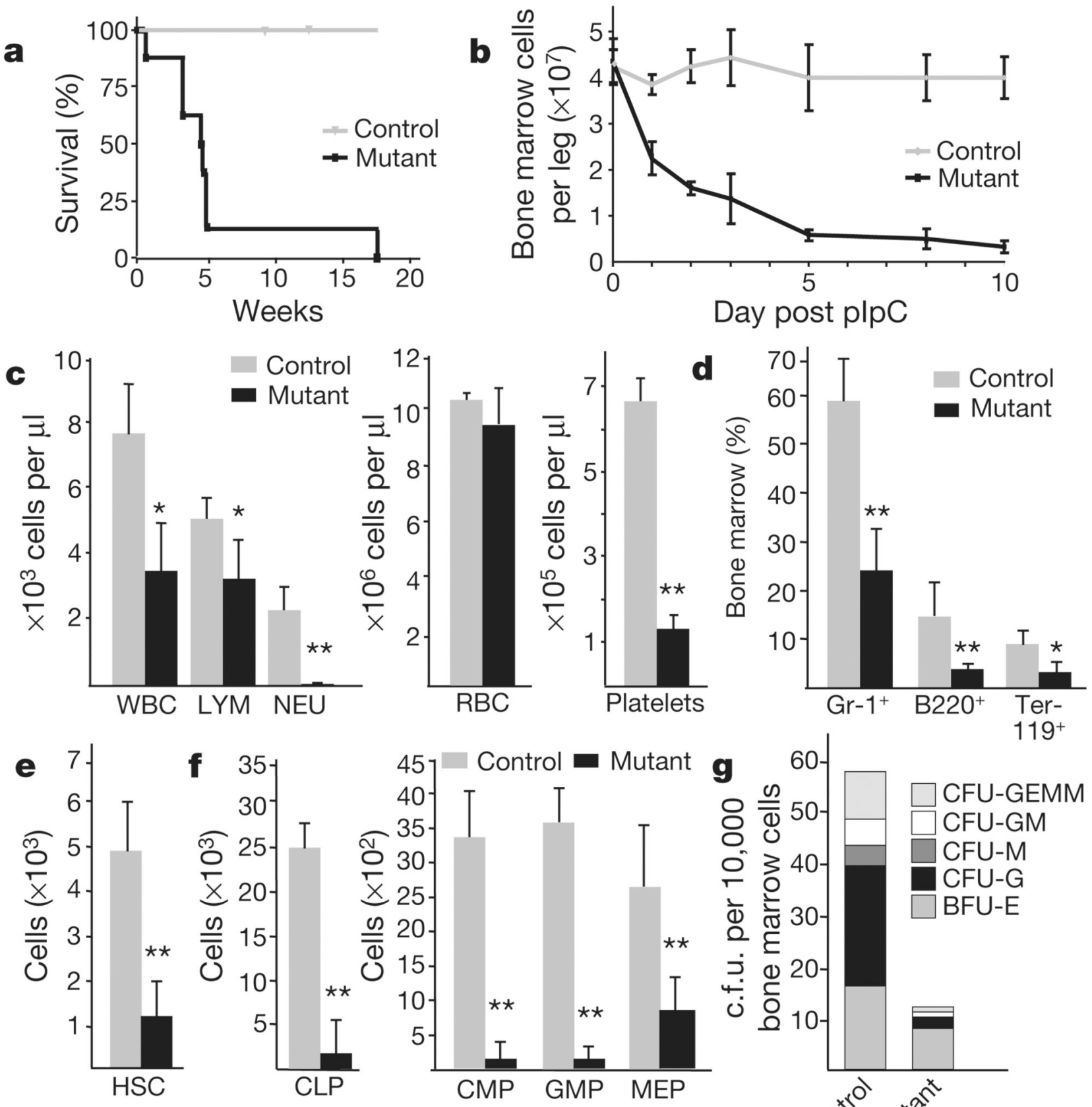

CMP

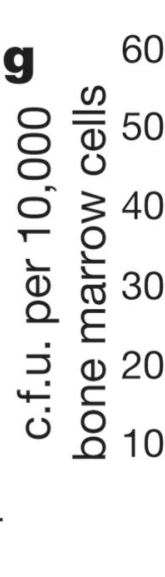

$119^{+}$

Figure 1. $L k b 1$ is required for haematopoiesis

Mutant (MxI-cre; $\left.L k b 1^{L / L}\right)$ and control (Mxl-cre; $L k b 1^{\mathrm{L} /+}$ or $\left.L k b 1^{L / L}\right)$ mice were injected with pIpC every second day over 7 days. a, Survival analysis; $n>10$ mice per genotype; $P<0.001$. b, Mononuclear bone marrow cellularity $(n=4)$. c-f, Analysis at day 5 for the indicated subpopulations ( $n=6$ mice per genotype). CLP, common lymphoid progenitors; CMP, common myeloid progenitors; GMP, granulocyte-macrophage progenitors; MEP, megakaryocyte-erythrocyte progenitors; NEU, neutrophil; RBC, red blood cell. g, CFU-C assay for myeloid progenitors. $* P<0.01 ; * * P<0.001$. Error bars in $\mathbf{b}-\mathbf{f}$ indicate mean \pm s.d. 

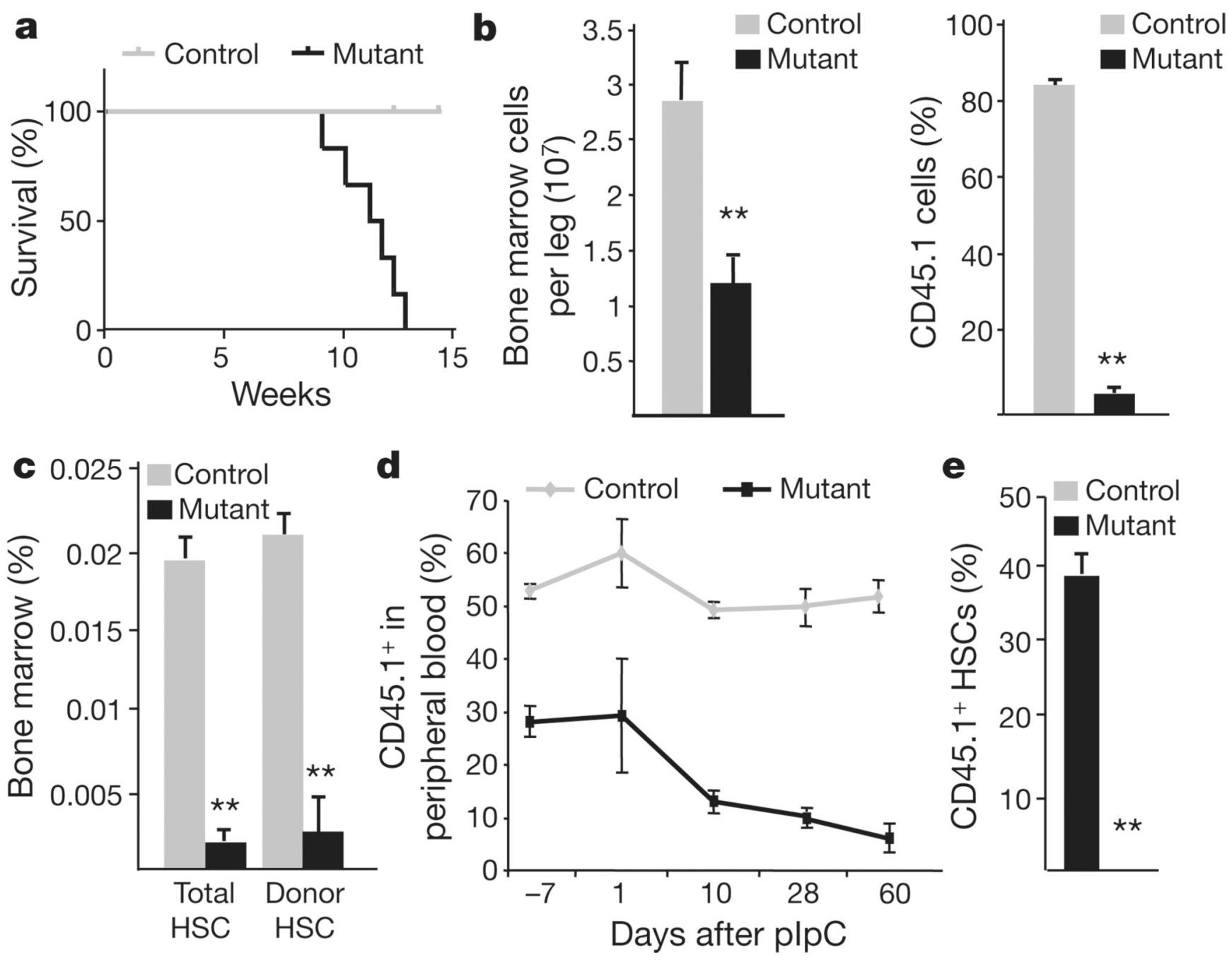

Figure 2. Cell-autonomous role of $L \mathrm{~kb} 1$ in haematopoiesis

a-c, After pIpC induction, transplanted mice were analysed for survival $(P<0.005)(\mathbf{a})$, bone marrow cellularity (left) and donor chimaerism (right) on day 5 (b), and total HSC numbers and donor contribution at day $18(\mathbf{c})$. d, e, Competitively transplanted mice were induced with pIpC and analysed for CD45.1 status in peripheral blood (d), and per cent CD45.1 HSCs at 4 weeks (e). $n>6$ mice per genotype in a-e. $* P<0.05$, $* * P<0.01$, all error bars indicate mean \pm s.d. 


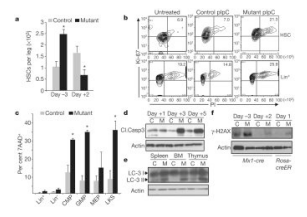

Figure 3. Impact of $L k b 1$ inactivation on proliferation and apoptosis

a, Quantification of HSCs in the Mxl-cre model at day -3 and day +2 after pIpC treatment. $n=3$ mice per genotype; $P<0.01$, error bars indicate mean \pm s.d. b, Cell-cycle analysis of HSCs (Ki-67/propidium iodide (PI) staining) at day $-3(n=3 ; P<0.01)$. c, Analysis viability in HSC and progenitor cells and $\mathrm{Lin}^{+}$cells at day 5 by 7AAD staining $(* P<0.001$, error bars indicate mean \pm s.d.). d-f, Immunoblot of control (C) and $L k b l$ mutant (M) mice for cleaved caspase-3 (Cl.Casp3) in bone marrow (BM) (d), LC3 in the indicated tissues at day 5 after pIpC (e), and phospho-H2AX levels in bone marrow (f). 
a

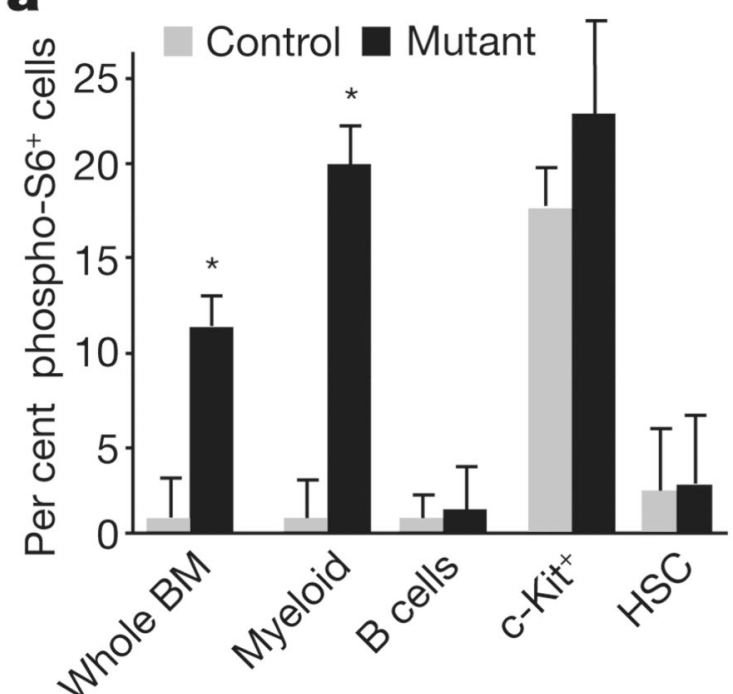

b

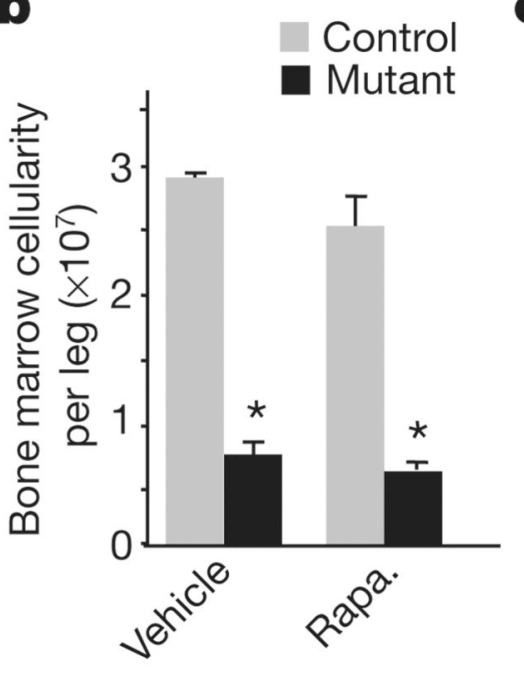

C

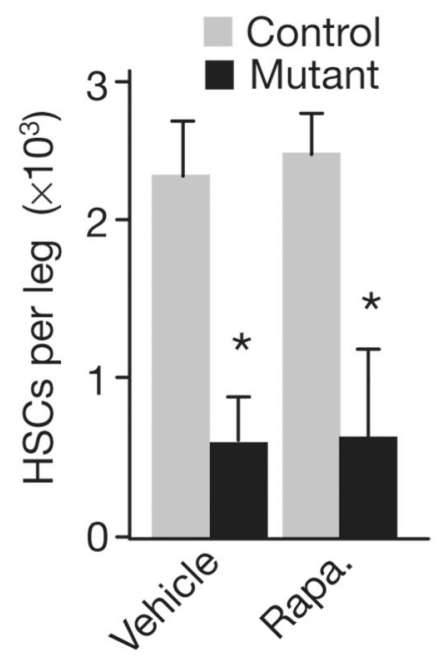

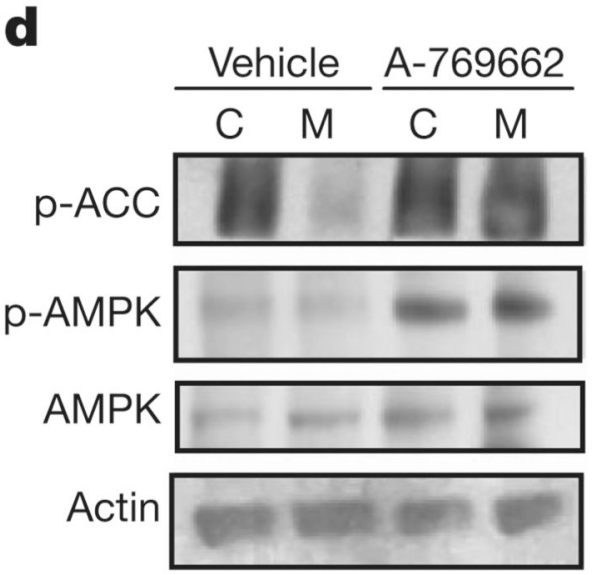
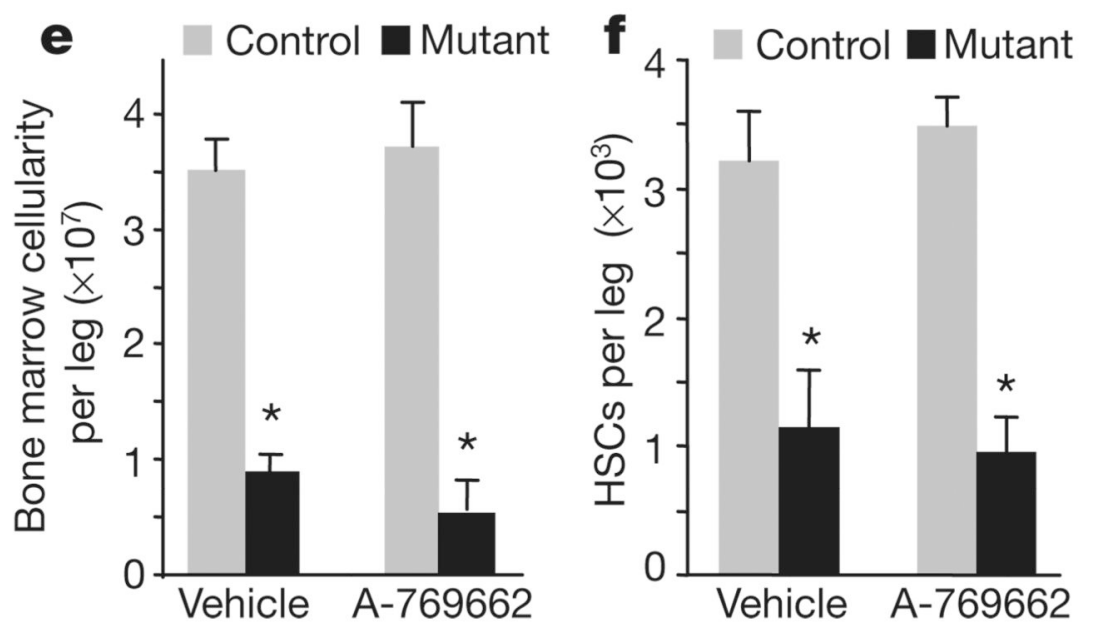

Figure 4. mTORC1 inhibition and AMPK activation do not rescue bone marrow failure in $\mathbf{L k b 1}$ mutants

a, Phospho(Ser 235/236)-S6 expression in bone marrow subpopulations at day 5 after pIpC treatment. b, c, Rapamycin (Rapa.) treatment does not rescue the drop in bone marrow cellularity (b) or HSCs (c) in $L k b l$ mutants at day 5 ( $n=4$ mice per genotype). d-f, The AMPK activator A-769662 restores phospho(Ser 79)-ACC levels in Lkbl mutant bone marrow cells $(\mathbf{d})$, yet does not rescue loss of bone marrow cellularity (e), or HSCs (f) at day $5(n=4)$. $* P<0.001$, all error bars indicate mean \pm s.d. 

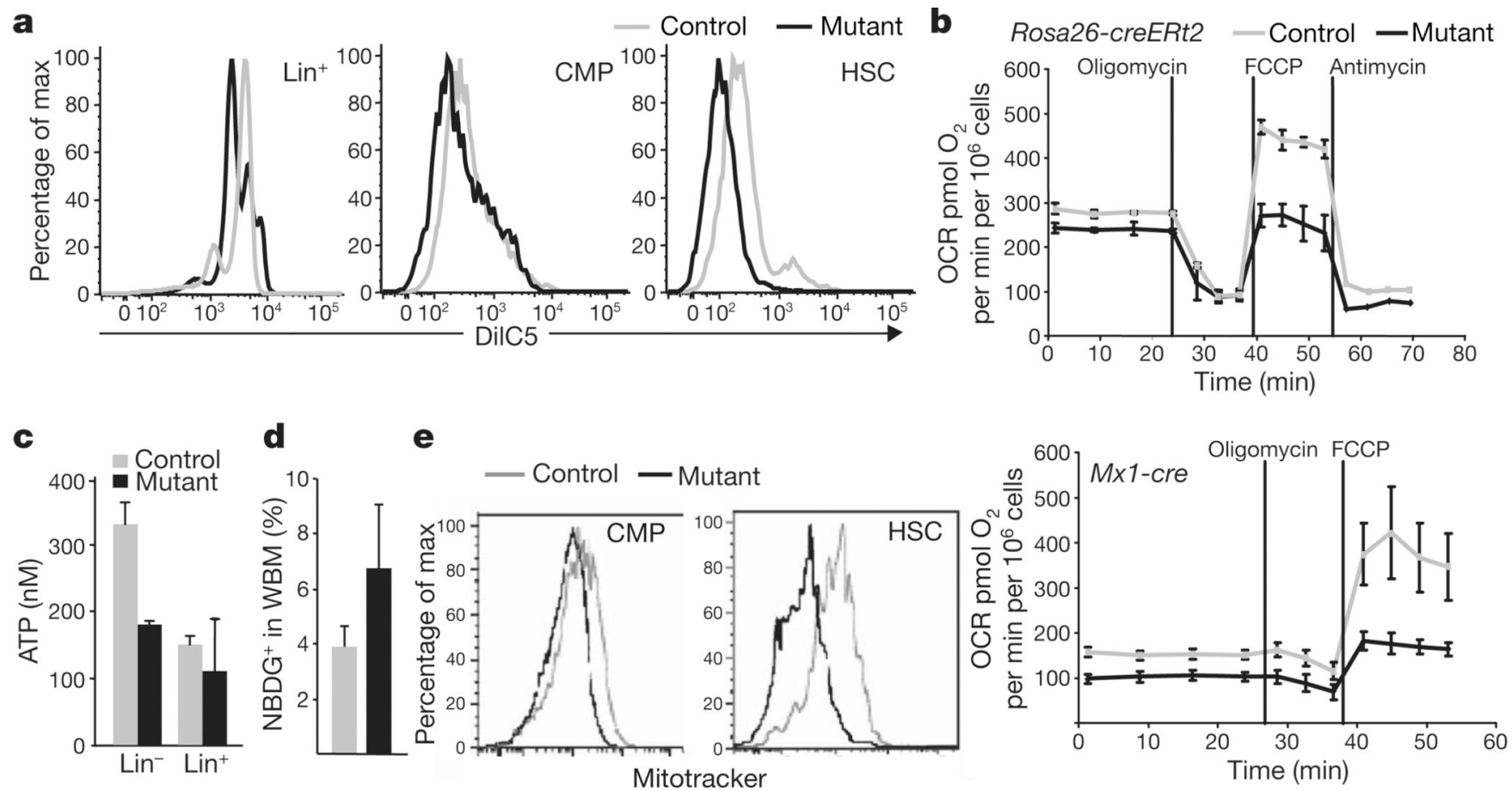

Figure 5. Inactivation of Lkb1 alters mitochondrial function of bone marrow cells a, Mitochondrial membrane potential of control and mutant cells at day 3 after pIpC assayed by DilC5 staining. b, Oxygen consumption rates (OCR) in control and mutant bone marrow cells under basal conditions and in response to $0.25 \mu \mathrm{M}$ oligomycin, $5 \mu \mathrm{M}$ fluoro-carbonyl cyanide phenylhydrazone (FCCP) or $1 \mu \mathrm{M}$ antimycin + rotenone at day 1 in the Rosa26creERt2 (top) and Mxl-cre (bottom) models. c, ATP levels of bone marrow cells at day 5 after pIpC. d, Glucose uptake in bone marrow at day 1. NBDG, fluorescent D-glucose analogue. e, Quantification of relative mitochondrial mass by Mitotracker staining (Mxl-cre model). $n=3$ mice per genotype; $P<0.001$; all error bars indicate mean \pm s.d. 